\title{
A superabsorbent polymer-containing wound dressing efficiently sequesters MMPs and inhibits collagenase activity in vitro
}

\author{
Cornelia Wiegand • Uta-Christina Hipler
}

Received: 6 March 2013/ Accepted: 14 June 2013/Published online: 25 June 2013

(c) The Author(s) 2013. This article is published with open access at Springerlink.com

\begin{abstract}
Superabsorbent polymer (SAP)-containing wound dressings present a valuable and unique category of wound management products. An in vitro approach was used to assess the effects of a new SAP dressing in treatment of non-healing wounds. It was shown that the SAP dressing possesses a significant binding capacity for MMP-2 and MMP-9 in vitro $(P<0.001)$. The inclusion of the bound proteases was so strong that no MMP-2 and only marginal amounts of MMP-9 were released from the dressing samples in a subsequent elution step. In addition, the SAP dressing was able to take up collagenase and reduce its activity in vitro. However, collagenase was not completely inactivated upon binding and enzyme-mediated substrate turnover could be observed at the dressings. In conclusion, in vitro data confirm the positive effect of the SAP wound dressing observed in vivo. The findings suggest that it should be specifically useful for highly exuding wounds with an elevated proteolytic activity that needs to be reduced to support healing.
\end{abstract}

\author{
Abbreviations \\ ECM Extracellular matrix \\ MMP Matrix metalloproteinase \\ PMN Polymorphonuclear \\ SAP Superabsorbent polymer \\ TIMP Tissue inhibitor of matrix metalloproteases
}

C. Wiegand $(\bowtie) \cdot$ U.-C. Hipler

Department of Dermatology, University Medical Center Jena,

Erfurter Str. 34, 07740 Jena, Germany

e-mail: c.wiegand@med.uni-jena.de

\section{Introduction}

Non-healing wounds, like venous, pressure and diabetic ulcer, have become a rising charge to society as an increasing number of patients suffer from wounds that fail to heal. Several studies have shown that exudates from these chronic wounds are characterized by elevated levels of matrix metalloproteinases (MMPs) [1, 2], contain excessive amounts of polymorphonuclear granulocyte-derived elastase (PMN elastase) [3-5], and high concentrations of free radicals [6]. These inflammatory mediators shift the balance of matrix synthesis and its degradation towards tissue destruction. The disproportionate action of proteases leads to considerable reduced amounts of growth factors [7] and proteinase inhibitors like tissue inhibitors of matrix metalloproteases [8]. Hence, modern wound management focuses on reduction of these inflammatory mediators and creation of a moist wound environment. Both should aid in establishing a physiological wound milieu and promote wound healing. For this purpose, a diversity of occlusive dressings is available in various forms, such as films, foams, or gels, made from different materials like alginates, polyurethane, hyaluronic acid, and collagen. However, highly exuding wounds may require other options as not all of these materials are able to handle an excess amount of exudate whose control is critical in the management of chronic wounds [9]. Therefore, wound dressings containing superabsorbent polymers (SAP) were devised. SAPs are able to take up a multiple amount of water of their own dry weight and are mainly utilized as an absorbent for water and aqueous solutions in diapers, adult incontinence products, feminine hygiene products, and similar applications [10]. They are mainly fabricated from acrylic acid and a crosslinker by solution or suspension polymerization [10]. The resulting polyacrylate superabsorbers have a high density of ionic 


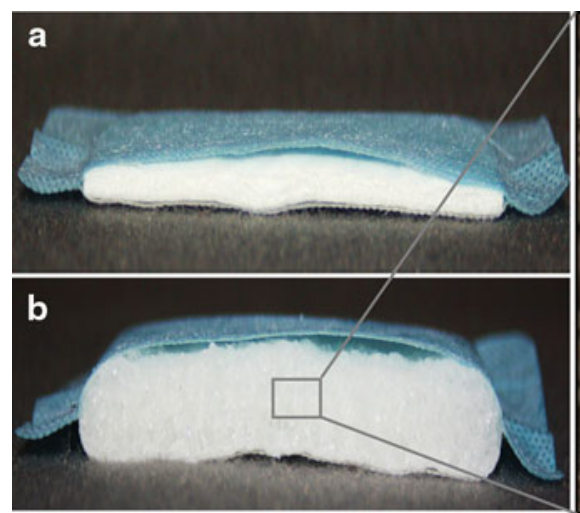

Fig. 1 The SAP-containing wound dressing consists of a 4-layer construction (a): wound contact layer, distribution layer, absorbent core, and a fluid repellent cover sheet (blue). Upon contact with fluid

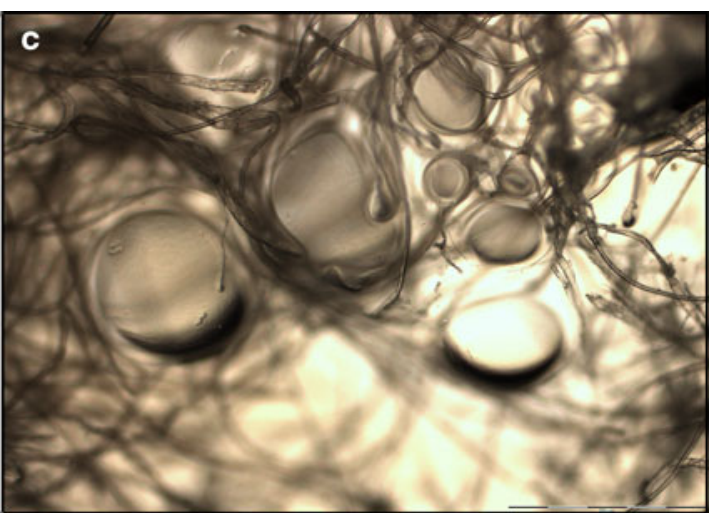

the absorbent core begins to swell (b). It is comprised of a cellulose fiber matrix enclosing the SAP particles (c, light microscopic image, $\times 20$ magnification)

$8 \mathrm{~mm}$ punch biopsies (Stiefel Laboratorium $\mathrm{GmbH}$, Germany) corresponding to $0.5 \mathrm{~cm}^{2}$. The samples were placed into 24-well cell culture plates (Greiner bio-one, Germany). Glass cover slips $\left(0.5 \mathrm{~cm}^{2}\right)$ were used as controls. Each specimen was taken in a final volume of $1 \mathrm{~mL}$ of protease solution. Samples were incubated up to $24 \mathrm{~h}$ at $37^{\circ} \mathrm{C}$ on a plate mixer (THERMOstar, BMG Labtech $\mathrm{GmbH}$, Germany). After incubation, supernatants were collected, immediately frozen and stored at $-20{ }^{\circ} \mathrm{C}$ until testing. Subsequently, bound protein was eluted from the individual wound dressing samples by shaking in $1 \mathrm{~mL}$ PBS for $1 \mathrm{~h}$ at $37{ }^{\circ} \mathrm{C}$. For determination of the MMP-2 and the MMP-9 concentrations, the specific ELISAs were purchased from R\&D Systems GmbH (Germany) and run as recommended by the manufacturer. Optical density was measured at $450 \mathrm{~nm}$ with a reference measurement at $620 \mathrm{~nm}$ using a plate photometer (BMG Labtech $\mathrm{GmbH}$, Germany). Subsequently, the protease concentrations were evaluated according to a 4-parameter-fit with lin-log coordinates for optical density (linear scale) and concentration (logarithmic scale).

\subsection{Inhibition of collagenase activity}

Lyophilized collagenase was reconstituted as recommended in the manufacturer's instructions (EnzChek Collagenase/Gelatinase assay kit, Invitrogen, Germany). A $1,000 \mathrm{U} / \mathrm{mL}$ stock solution of collagenase was prepared in distilled water. For experiments, the stock solution was diluted to $0.2 \mathrm{U} / \mathrm{mL}$ in the reaction buffer $(0.1 \mathrm{M}$ Tris$\mathrm{HCl}, \mathrm{pH} 8.0$, containing $0.2 \mathrm{mM}$ sodium azide and $0.5 \%$ bovine serum albumin). Wound dressing samples were cut using $8 \mathrm{~mm}$ punch biopsies (Stiefel Laboratorium $\mathrm{GmbH}$, Germany) corresponding to $0.5 \mathrm{~cm}^{2}$. The samples were placed into 24-well cell culture plates (Greiner bio-one, Germany). Glass cover slips $\left(0.5 \mathrm{~cm}^{2}\right)$ were used as 
controls. Each specimen was taken in a final volume of $1 \mathrm{~mL}$ of protease solution. Samples were incubated up to $24 \mathrm{~h}$ at $37{ }^{\circ} \mathrm{C}$ on a plate mixer (THERMOstar, BMG Labtech $\mathrm{GmbH}$, Germany). After incubation, supernatants were collected, immediately frozen and stored at $-20{ }^{\circ} \mathrm{C}$ until testing. Subsequently, bond protein was eluted from the individual wound dressing samples by shaking in $1 \mathrm{ml}$ PBS for $1 \mathrm{~h}$ at $37^{\circ} \mathrm{C}$. Collagenase activity was determined using the Collagenase/Gelatinase assay kit (Invitrogen, Germany). The assay was run as recommended in the instructions. Briefly, $80 \mu \mathrm{L}$ reaction buffer and $20 \mu \mathrm{L}$ DQ gelatin substrate were added, followed by the injection of $100 \mu \mathrm{L}$ sample. The fluorescence was measured continuously for $1 \mathrm{~h}$ at room temperature using a fluorescence plate reader (FLUOstar Galaxy, BMG Labtech; excitation wavelength: $495 \mathrm{~nm}$, emission wavelength: $538 \mathrm{~nm}$ ). Additionally, eluted dressing samples were incubated for $1 \mathrm{~h}$ with $800 \mu \mathrm{L}$ reaction buffer and $200 \mu \mathrm{L}$ DQ gelatin. Afterwards, $200 \mu \mathrm{L}$ samples were transferred to a black 96-well plate and substrate turnover was measured by fluorescence intensity (excitation wavelength: $495 \mathrm{~nm}$, emission wavelength: $538 \mathrm{~nm}$ ) using a fluorescence plate reader (FLUOstar Galaxy, BMG Labtech).

\subsection{Statistical analysis}

All values cited are expressed as means $\pm \mathrm{SE}$ (standard error). One-way analysis of variance was carried out to determine statistical significances (Microsoft $^{\circledR}$ Excel 2000). Differences were considered statistically significant at a level of $P<0.05$. Asterisks indicate significant deviations from the control at the respective incubation time $(* P<0.05 ; * * P<0.01 ; * * * P<0.001)$.

\section{Results}

The SAP dressing tested exhibited a significant binding capacity for MMP-2 in vitro $(P<0.001$; Fig. $2 \mathrm{a})$. The dressing samples quickly reduced the amount of MMP-2 in the supernatant upon contact. Moreover, no protein was detected in the eluate (Fig. 2c). It was observed that the protease was so tightly bound by the SAP dressing samples that even disruption of the dressing and aggressive elution techniques (e.g. vortexing or ultrasonic bath) did not lead to the release of MMP-2 in vitro. However, bound MMP-2 could be perceived by direct incubation of the disrupted dressing samples with the respective ELISA antibodies (Fig. 3a). Moreover, these samples were subjected to light microscopy, where binding of MMP-2 was verified by the blue color of the TMB-substrate (Fig. 3b). It could be shown that MMP-2 is bound to the cortical zone of the polyacrylate particles. In addition, the SAP dressing was found to significantly bind MMP-9 in vitro $(P<0.001$; Fig. 2b). Only a marginal amount of the protease was released from the dressings in the subsequent elution step
Fig. 2 The SAP-containing wound dressing reduces the MMP-2 (a) and MMP-9

(b) concentration in a defined enzyme solution. No MMP-2 was detected in the eluate (c). Only marginal amounts of MMP-9 could be eluted from the samples (d)
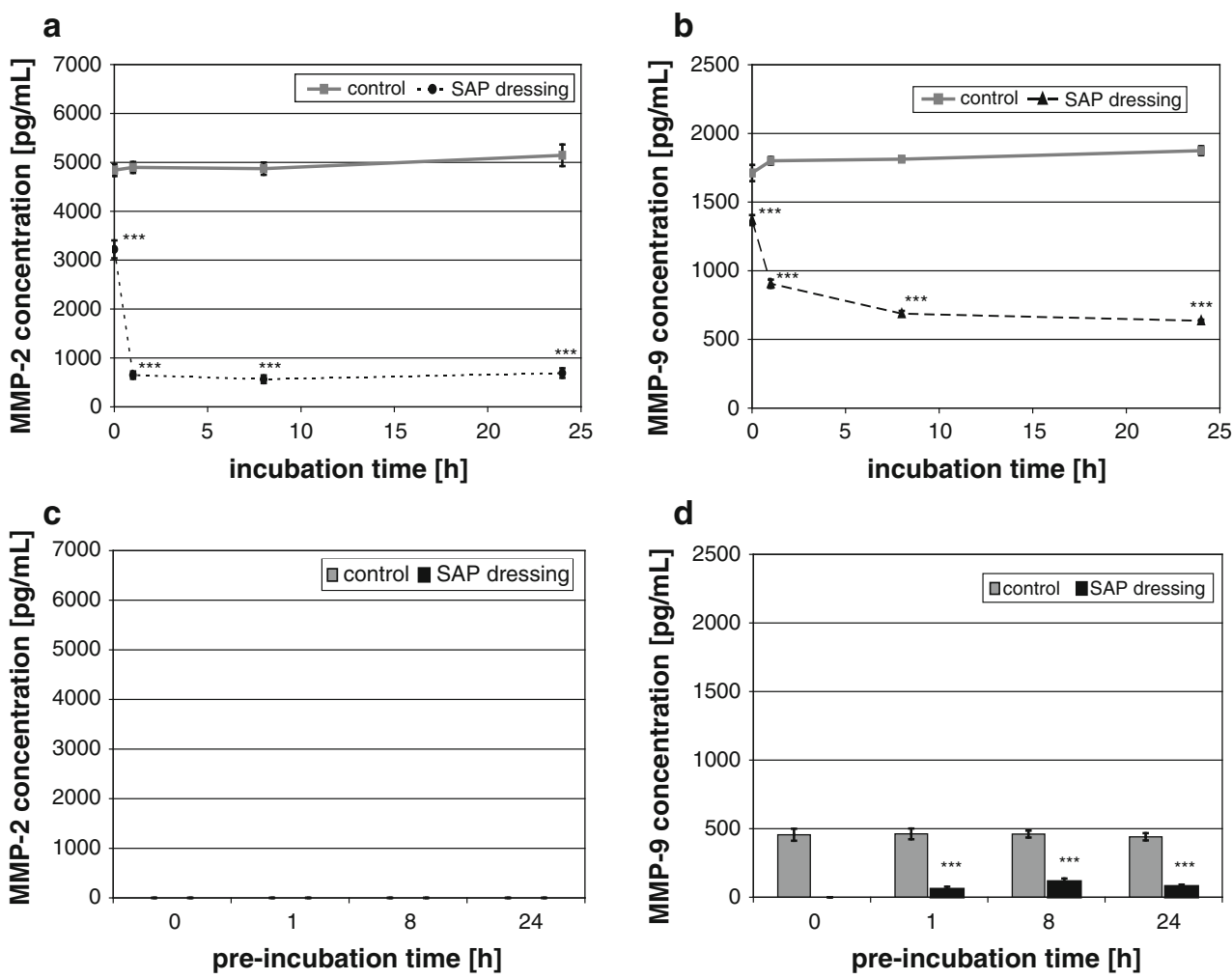

d

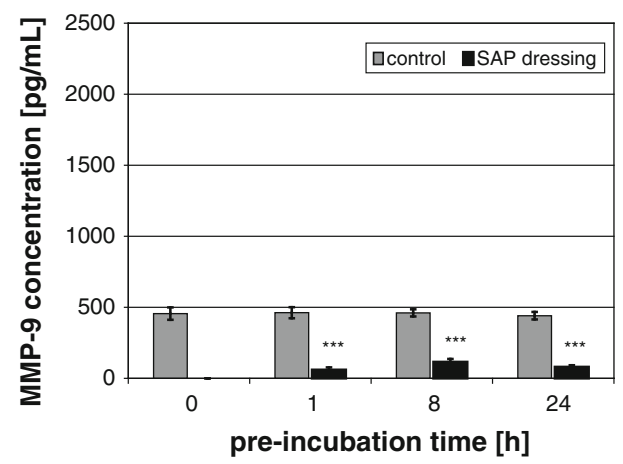


Fig. 3 MMP-2 could be detected in the disrupted dressing samples by direct incubation with the respective ELISA antibodies (a). Light microscopy of the samples $(\times 20$ magnification) revealed that MMP-2 is bound to the cortical zone of the particles, distinguishable by the light blue color development (b)

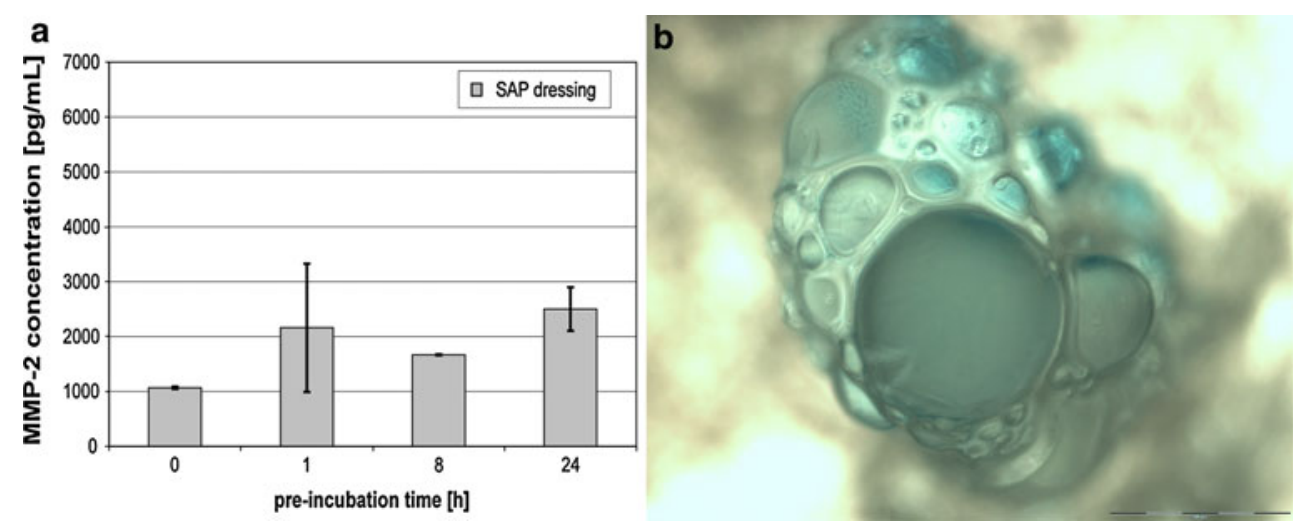

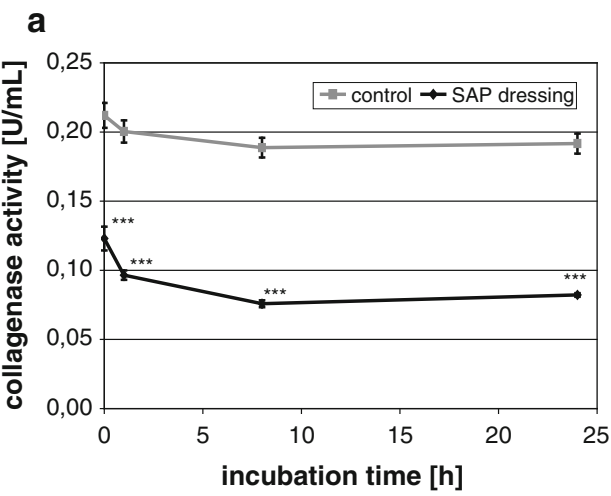

Fig. 4 Collagenase activity was significantly reduced by the SAP dressing (a). No collagenase activity was observed in the eluate. However, collagenase was not inactivated by binding to the SAP;

compared to the control (Fig. 2d). Moreover, a significant effect of the SAP dressing on the activity of collagenase in the supernatant was observed $(P<0.001$; Fig. $4 a)$. Almost no enzyme activity was detectable in the eluate (data not shown). However, it could be demonstrated that the collagenase bound to the dressing samples remains at least partially active (Fig. 4b). An enzyme-mediated substrate turnover could be observed directly at the SAP beads of the dressing (Fig. 5).

\section{Discussion}

Exudate control is crucial in management of chronic wounds [9]. The excessive fluid needs to be removed from the wound (liquid acquisition), quickly dispersed in the dressing (liquid distribution), and maintained inside the material (liquid retention). The highest liquid retention can be achieved with cross-linked, high molecular-weight polyelectrolytes, called SAPs [11]. Most SAPs are cross-linked networks of flexible polymer chains with abundant ionizable carboxyl groups,

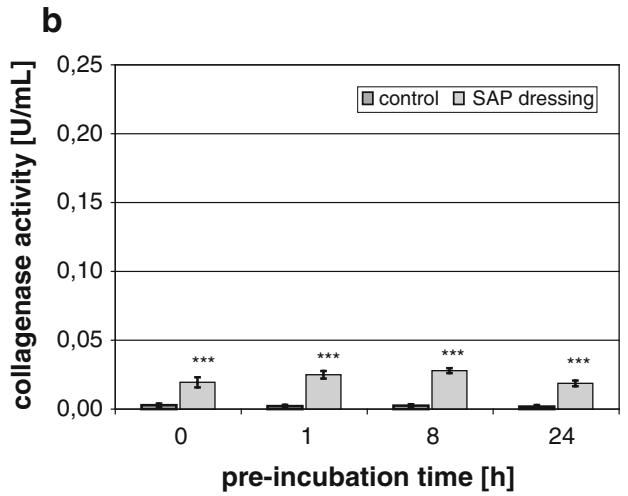

a significant substrate turnover could be noted when the dressings were incubated with the collagenase substrate DQ gelatin (b)

typically joined with sodium ions [10,11]. Fluid absorption occurs through osmotic pressure caused by the concentration gradient of electrolytes inside and outside the SAP particles [11]. This process is accompanied by the detachment of the sodium ion, which leaves the charged carboxyl group behind [10], and the unfolding of the macromolecule chains, due to the repulsion forces of the negatively charged sites [11]. This allows the SAP to swell and absorb large amounts of liquid. During this process, SAPs also take up and retain proteins, cell debris and even micro-organisms [12-15]. Binding of proteins is most likely due to electrostatic forces that arise between their positively charged groups and the negatively charged carboxyl groups of the polyacrylate. It seems likely that the SAP dressing tested binds and removes MMPs from the test solution by the same mechanism, e.g. Eming et al. [13] observed in their experiments a significant reduction of MMP concentration. Moreover, SAPs have been shown to reduce the concentration of PMN elastase by electrostatic interactions [12]. Likewise, MMPs are known to be bound to other polymers, such as oxidized regenerated cellulose, collagen or alginate, 

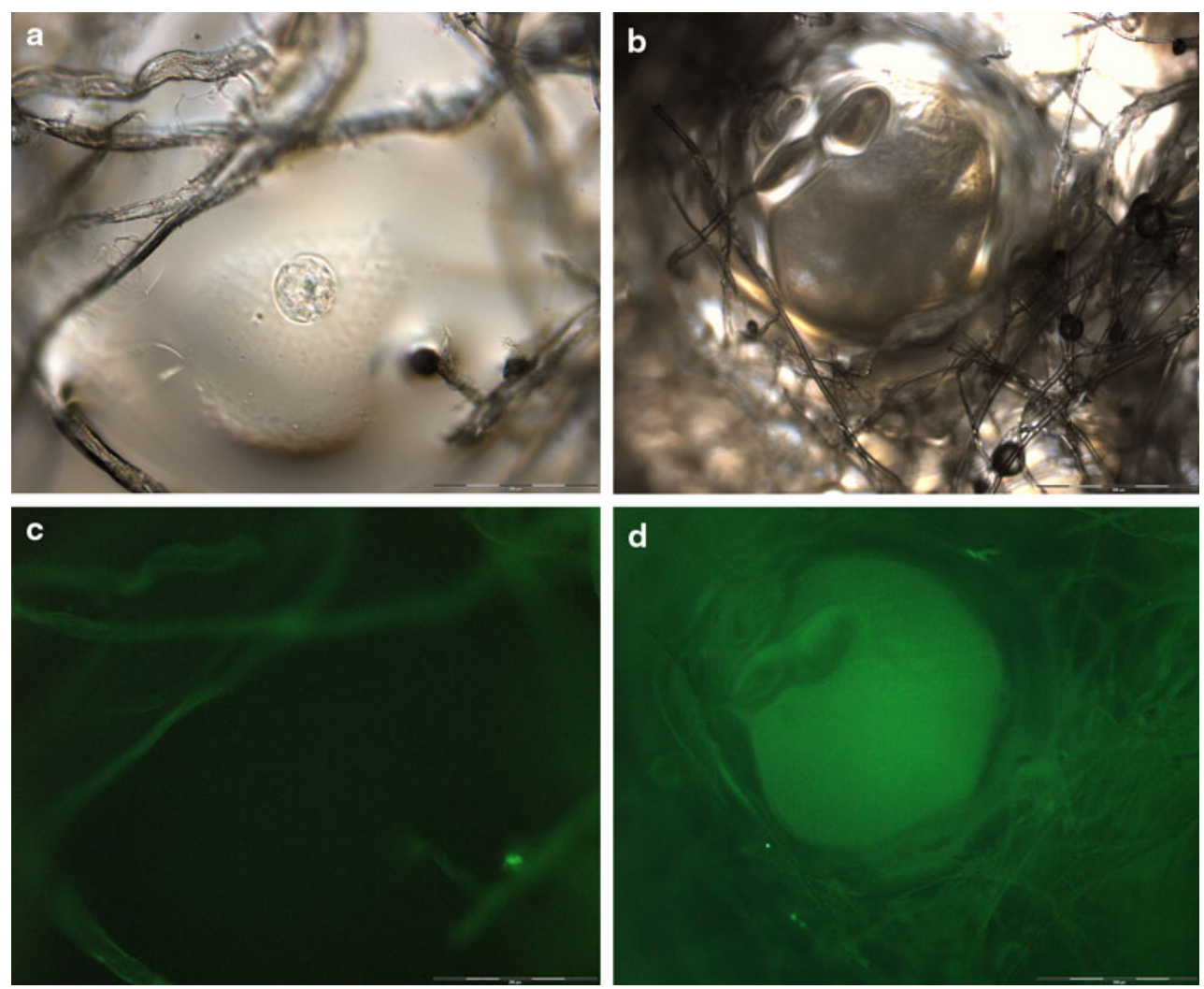

Fig. 5 Light microscopic images of the SAP dressing show the fibers and fluid swollen polyacrylate particles of the absorbent layer $(\mathbf{a}, \mathbf{b})$. The fibers exhibit a slight intrinsic fluorescence but the polyacrylate particle is not visible in fluorescence imaging (ex: $485 \mathrm{~nm}$, em: $520 \mathrm{~nm})(\mathbf{c})$. Polyacrylate particles with bound collagenase became

by electrostatic forces [16-18]. In accordance, it was found that the SAP dressing tested exhibited a significant binding capacity for MMP-2 and MMP-9 in vitro. Most remarkably was the fast kinetic of the attachment of the protease to the SAP, upon contact the enzyme concentration in solution was decreased. Moreover, binding of MMP-2 was found to be irreversible and only marginal amounts of MMP-9 could subsequently be eluted from the material. These results are similar to the outcome of a recently published study that demonstrated the binding of MMP-2 as well as MMP-9 and the inactivation of collagenase by a superabsorbant dressing [19]. Here, it could further be demonstrated that the proteases are bound to the cortical zone of the SAP particles in the absorbent layer of the dressing. These findings are in line with previous reports by Eming et al. [13]. Additional experiments revealed that incubation with the SAP dressing reduces collagenase activity by sequestration and removal of the protease from the fluid in vitro. However, collagenase activity was not inhibited as the protease remained at least partially active when bound to the dressing's core. Comparable results were observed for binding of elastase by other SAP-containing dressings that were able to take up elastase and effectively prevent its subsequent release but were not visible in fluorescence imaging (ex: $485 \mathrm{~nm}$, em: $520 \mathrm{~nm}$ ) after incubation with collagenase substrate (d). Hence, it can be concluded that the SAP beads in the dressing bind the active enzyme and efficiently remove it from the solution, but collagenase remained at least partially active. $(\times 20$ magnification)

capable to impede enzyme-mediated substrate turnover [12]. In contrast, Eming et al. [13] observed in their experiments also an indirect effect of SAP on the MMP activity by binding of essential $\mathrm{Ca}^{2+}$ and $\mathrm{Zn}^{2+}$ ions.

\section{Conclusion}

It can be expected that the SAP dressing achieves a beneficial effect on wound healing not only by absorbing exudate but also by reducing the concentration of proteases such as MMP-2 and MMP-9. Hence, it should be particularly effective in the cleansing phase during treatment of chronic wounds where the proteolytic activity is high and tissue destruction occurs.

Acknowledgments This work was funded by Molnlycke Healthcare Group (UK). The authors would like to thank Denise Reichmann for excellent technical assistance.

Open Access This article is distributed under the terms of the Creative Commons Attribution License which permits any use, distribution, and reproduction in any medium, provided the original author(s) and the source are credited. 


\section{References}

1. Wysocki AB, Staiano-Coico L, Grinnel F. Wound fluid from chronic leg ulcers contains elevated levels of metalloproteinases MMP-2 and MMP-9. J Invest Dermatol. 1993;101:64-8.

2. Trengove NJ, Stacey MC, Macauley S, Bennett N, Gibson J, Burslem F, Murphy G, Schultz G. Analysis of the acute and chronic wound environments: the role of proteases and their inhibitors. Wound Rep Reg. 1999;7:442-52.

3. Yager DR, Nwomeh BC. The proteolytic environment of the chronic wounds. Wound Rep Reg. 1999;7:433-41.

4. Herrick S, Ashcroft G, Ireland G, Horan M, McCollum Ch, Ferguson M. Up-regulation of elastase in acute wounds of healthy aged humans and chronic venous leg ulcers are associated with matrix degradation. Lab Invest. 1997;77:281-8.

5. Barrick B, Campbell EJ, Owen CA. Leukocyte proteinases in wound healing: roles in physiologic and pathologic processes. Wound Rep Reg. 1999;7:410-22.

6. Rojkind M, Dominguez-Rosales J-A, Nieto N, Greenwel P. Role of hydrogen peroxide and oxidative stress in healing responses. Cell Mol Life Sci. 2002;59:1872-91.

7. He C, Hughes MA, Cherry GW, Arnold F. Effects of chronic wound fluid on the bioactivity of platelet-derived growth factor in serum-free medium and its direct effect on fibroblast growth. Wound Rep Reg. 1999;7:97-105.

8. Chen SM, Ward SI, Olutoye OO, Diegelmann RF, Cohen IK. Ability of chronic wound fluids to degrade peptide growth factors is associated with increased levels of elastase activity and diminished levels of proteinase inhibitors. Wound Rep Reg. 1997;5:23-32.

9. Faucher N, Safar H, Baret M, Philippe A, Farid R. Superabsorbent dressings for copiously exuding wounds. Br J Nurs. 2012; 21:S26-8.

10. Pytlik E, Molino D, Moritz J. Superabsorbent Polymers (SAP). In: Introduction to polymers. UB Engineering-University at
Buffalo. 2005. http://www.eng.buffalo.edu/courses/ce435/Diapers/ Diapers.html. Accessed 6 Mar 2013.

11. Dutkiewicz JK. Superabsorbent materials from shellfish waste-a review. J Biomed Mater Res. 2002;63:373-81.

12. Wiegand C, Abel M, Ruth P, Hipler UC. Superabsorbent polymercontaining wound dressings have a beneficial effect on wound healing by reducing PMN elastase concentration and inhibiting microbial growth. J Mater Sci Mater Med. 2011;22:2583-90.

13. Eming S, Smola H, Hartmann B, Malchau G, Wegner R, Krieg T, Smola-Hess S. The inhibition of matrix metalloproteinase activity in chronic wounds by a polyacrylate superabsorber. Biomaterials. 2008;29:2932-40.

14. Paustian C, Stegman MR. Preparing the wound for healing: the effect of activated polyacrylate dressing on debridement. Ostomy Wound Manage. 2003;49:9.

15. Mahr R. The mode of action of a superabsorbent polymer wound dressing (TenderWet ${ }^{\circledR}$ ). Ostomy Wound Manage. 2003;49:2A.

16. Wiegand $\mathrm{C}$, Heinze T, Hipler UC. Comparative in vitro study on cytotoxicity, antimicrobial activity, and binding capacity for pathophysiological factors in chronic wounds of alginate and silver-containing alginate. Wound Rep Reg. 2009;17:511-21.

17. Schönfelder U, Abel M, Wiegand C, Klemm D, Elsner P, Hipler UC. Influence of selected wound dressings on PMN elastase in chronic wound fluid and their antioxidative potential in vitro. Biomaterials. 2005;26:6664-73.

18. Wiegand C, Abel M, Ruth P, Wilhelms T, Schulze D, Norgauer J, Hipler UC. Effect of the sterilization method in the performance of collagen type I on chronic wound parameters in vitro. J Biomed Mater Res B. 2009;90B:710-9.

19. Wiegand C, White RJ. Binding and inhibition of protease enzymes, including MMPs, by a superabsorbent dressing in vitro. J Wound Care. 2013;22:221-7. 